\title{
Frequency optimisation of composite cylinder using an evolutionary algorithm and neural networks
}

\author{
Bartosz Miller ${ }^{1, *}$ and Leonard Ziemiański ${ }^{1}$ \\ ${ }^{1}$ Rzeszów University of Technology, Faculty of Civil and Environmental Engineering and Architecture
}

\begin{abstract}
The paper deals with the optimisation of dynamic properties of a composite cantilever cylinder. The optimised parameters are both the fundamental natural frequency $f_{1}$ as well as the gap in frequency space around a selected external excitation force allowing to avoid the resonance phenomenon. The optimisation is performed using a novel approach combining particle swarm optimisation and artificial neural networks. The evolutionary algorithms are used to solve the optimisation problem with many local minima while neural networks are used to substitute time-consuming finite element calculations of the minimisation problem objective function.
\end{abstract}

\section{Introduction}

Multilayered fibre-reinforcement composite materials are nowadays widely applied in many engineering structures, before all — but not only — in aircraft and automotive manufacturing. The composite structures are durable and reliable, unfortunately, their dynamic behaviour is not as widely studied and tested as in case of structures made of traditional materials (e.g. steel). The slenderness and low mass of constructions made of multilayered composites may be the reason for a different behaviour of such structures and therefore should be carefully studied.

The problem studied in this work is related to the necessity to avoid resonance phenomenon in structures subjected to an external load with a priori known excitation frequency $p$. Resonance, appearing when the external excitation frequency $p$ is equal (or very similar) to any of analysed structure's natural frequencies $f_{i}$, may be avoided either by maximisation of the fundamental natural frequency (assuming that the excitation frequency is low enough) or by creating a gap among all natural frequencies around the excitation frequency (when it is too high to fit below fundamental natural frequency).

The optimisation problem of maximisation of $f_{1}$ or creating the maximal gap around $p$ is herein solved using one of the evolutionary algorithms, namely Particle Swarm Optimisation (PSO) (see [2]). The evolutionary algorithm has been chosen since the optimisation problem is non-convex, with a number of local minima, and may be rather difficult to be solved using traditional optimisation algorithms. Moreover, the values of the objective function being minimised are not computed using the usual approach involving Finite Element Method (FEM). The approach involving FEM, though very precise and reliable, is extremely time-consuming when using evolutionary algorithms (including PSO) that need to repeatedly calculate the

\footnotetext{
*e-mail: bartosz.miller@prz.edu.pl
} 
value of the objective function. Artificial Neural Networks (ANN) (see [3, 4]) are applied instead of FEM since properly trained and tested ANN is able to give the objective function value almost instantly.

The proposed optimisation procedure uses the fact that in the multilayered composites structures it is possible to control, to a certain extent, the dynamic parameters by only changing the lamination angles (see [5]). Therefore, the control variables are the data describing the fibre angles in each of the composite layers (so-called stacking sequence), while the optimised values are either $f_{1}$ or the width of the gap around excitation frequency $p$.

\section{The investigated model and the proposed optimisation procedure}

\subsection{The investigated model}

The investigated model is a thin-walled cylinder fixed at one end, the length of the cylinder is $l=6 \mathrm{~m}$, the diameter $R=0.6103 \mathrm{~m}$ and the wall thickness $t=16 \mathrm{~mm}$. The cylinder walls are made from multilayered fibre-reinforcement composite material with the properties taken from [6]: $E_{1}=141.9 \mathrm{GPa}, E_{2}=9.78 \mathrm{GPa}, v_{12}=0.42, G_{12}=6.13 \mathrm{GPa}$ and $\rho=$ $1445 \mathrm{~kg} / \mathrm{m}^{3}$. The finite element model of the thin-walled cylinder (see fig. 1) is built in commercial FEM code Adina [1] using multi-layered shell 4-node MITC4 elements (first order shear theory), the number of which equals 80 along the circumferential direction and 120 in the axial direction. The overall number of FE elements, nodes and degrees of freedom equals 6900, 9680 and 58080, respectively. The number of composite layers $n$ in cylinder wall is equal to eight.

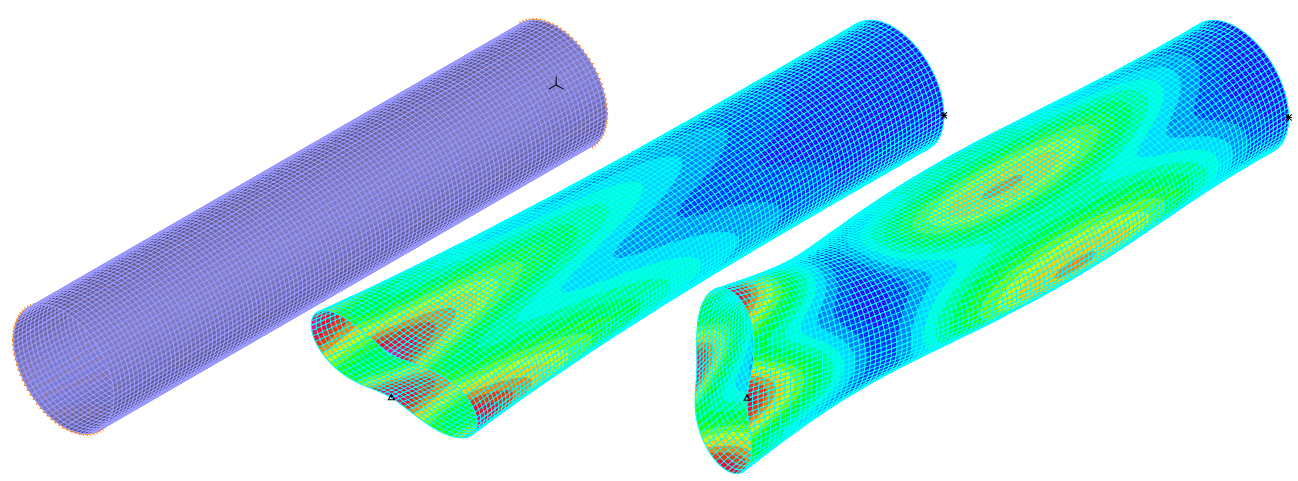

Figure 1. FE model of the thin-walled cylinder, the first and the fifth mode shapes

\subsection{The optimisation problem}

The optimisation tasks to be performed, namely the maximisation of the fundamental frequency $f_{1}$ or the maximisation of the gap around excitation frequency $p$, may be described as:

$$
\boldsymbol{\Lambda}^{*}=\underset{\boldsymbol{\Lambda} \in \mathbb{L}^{8}}{\arg \min }\{\mathrm{g}(\boldsymbol{\Lambda})\}
$$

where $\boldsymbol{\Lambda}$ is a stacking sequence vector describing composite lamination angles $\boldsymbol{\Lambda}=$ $\left\{\lambda_{1}, \lambda_{2}, \ldots, \lambda_{8}\right\}, \mathbb{L}^{8}$ is an 8-dimensional space of possible values of $\boldsymbol{\Lambda}$ and $g(\boldsymbol{\Lambda})$ is an objective function to be minimised:

$$
\mathrm{g}(\boldsymbol{\Lambda})=-f_{1}(\boldsymbol{\Lambda})
$$


for maximisation of $f_{1}$, where $f_{1}(\boldsymbol{\Lambda})$ shows a relation between the fundamental frequency $f_{1}$ and the stacking sequence $\boldsymbol{\Lambda}$, and

$$
\mathrm{g}(\boldsymbol{\Lambda})=-\min _{i=\{1, \ldots, 10\}}\left|f_{i}(\boldsymbol{\Lambda})-p\right|
$$

for maximisation of the frequency gap around excitation frequency $p$ (where $|\cdot|$ indicates the absolute value). The optimisation tasks discussed herein can be described by the following formulas:

$$
\mathbf{\Lambda}^{*}=\underset{\Lambda \in \mathbb{L}^{8}}{\arg \min }\left\{-f_{1}(\boldsymbol{\Lambda})\right\}
$$

for maximisation of $f_{1}$ and

$$
\mathbf{\Lambda}^{*}=\underset{\Lambda \in \mathbb{L}^{8}}{\arg \min }\left\{-\min _{i=\{1, \ldots, 10\}}\left|f_{i}(\mathbf{\Lambda})-p\right|\right\}
$$

for maximisation of the frequency gap around excitation frequency $p$. The values of the objective function are in this paper calculated using ANN, the Eq. 4 can be given in a form including the ANN application:

$$
\boldsymbol{\Lambda}^{*}=\underset{\Lambda \in \mathbb{L}^{8}}{\arg \min }\left\{-\mathrm{ANN}_{1}(\boldsymbol{\Lambda})\right\}
$$

Similarly, Eq 5 can be given as:

$$
\boldsymbol{\Lambda}^{*}=\underset{\Lambda \in \mathbb{L}^{8}}{\arg \min }\left\{-\min _{i=\{1, \ldots, 10\}}\left|\operatorname{ANN}_{i}(\mathbf{\Lambda})-p\right|\right\}
$$

where $\operatorname{ANN}_{i}(\mathbf{\Lambda})$ is the value of the $i^{\text {th }}$ natural frequency calculated by ANN for the lamination angles set $\mathbf{\Lambda}$.

To get an ANN properly approximating the FEM solution:

$$
\operatorname{ANN}(\boldsymbol{\Lambda}) \cong \operatorname{FEM}(\boldsymbol{\Lambda})
$$

a number of vector pairs (called patterns), composed of $\boldsymbol{\Lambda}^{j}$ and $\mathbf{f}^{j}=\left\{f_{1}^{j}, f_{2}^{j}, \ldots, f_{10}^{j}\right\}=$ $\operatorname{FEM}(\boldsymbol{\Lambda})$, have to be prepared. Most of the pairs $\left(\boldsymbol{\Lambda}^{j}, \mathbf{f}^{j}\right)$ create a learning set applied to tune the ANN parameters, the others create a testing set applied to check the accuracy of the trained ANN.

\section{The results of the optimisation}

The first step in the proposed procedure is to teach neural networks to predict the dynamic properties of the considered structure. Supervised learning of the ANN requires, in turn, the preparation of a set of patterns. The patterns are generated using FEM for different combinations of lamination angles, 12, 664 of them create the learning set and 8,000 create the testing set. The overall number of FE calls necessary to create both pattern sets should be considered together with the following values:

- 20,664 - the number of FE calls in case a novel PSO+ANN procedure described in this paper is applied,

- 160,000 - the estimated number of FE calls in case classical optimisation procedure (with neither PSO nor ANN involved) is applied; the estimation is obtained on the basis of the number if FE calls during the maximisation of $f_{1}$ performed using classical approach, 
- 2, 800,000 - the estimated number of FE calls during the PSO optimisation involving FEM (not ANN) to compute the objective function values; the estimation is obtained as a simple multiplication (the swarm size: 100; the average number of PSO iterations: 80 ; each procedure repeated 50 times; 7 optimisation cases: maximisation of $f_{1}$, maximisation the gap around $p=30 \mathrm{~Hz}, 40 \mathrm{~Hz}, 50 \mathrm{~Hz}, 60 \mathrm{~Hz}, 70 \mathrm{~Hz}$ and $80 \mathrm{~Hz}$ ).

In case of PSO+FEM procedure, the legitimacy of using ANN is obvious because the number of FE calls is reduced by two orders of magnitude. In case of the application of classical methods, the number of FE calls is higher than for PSO+ANN procedure by about 8 times, the time consumption will increase significantly since the trained ANN gives its approximation almost instantly while FEM needs at least a dozen of seconds to calculate the desired result.

The neural networks are trained to give the values of the first ten natural frequencies of the composite cylinder for a given set of lamination angles which allows calculating the objective function values almost instantly (see Eq. 2 and Eq. 3). Once the ANN is ready the main PSO optimisation starts.

Table 1. Maximisation of the distance between any natural frequency $f_{i}$ and excitation frequency $p$

\begin{tabular}{ccc}
\hline $\begin{array}{c}\text { External } \\
\text { excitation } \\
\text { frequency }\end{array}$ & $\begin{array}{c}\text { Minimal distance to } f_{i} \\
\text { absolute } \\
\text { value }\end{array}$ & $\begin{array}{c}\text { relative } \\
\text { value }\end{array}$ \\
\hline$<30 \mathrm{~Hz}$ & maximal $f_{1}=44.99 \mathrm{~Hz}$ \\
$30 \mathrm{~Hz}$ & $12.9 \mathrm{~Hz}$ & $23.6 \%$ \\
$40 \mathrm{~Hz}$ & $13.5 \mathrm{~Hz}$ & $33.6 \%$ \\
$50 \mathrm{~Hz}$ & $20.8 \mathrm{~Hz}$ & $41.5 \%$ \\
$60 \mathrm{~Hz}$ & $23.7 \mathrm{~Hz}$ & $39.5 \%$ \\
$70 \mathrm{~Hz}$ & $24.0 \mathrm{~Hz}$ & $34.3 \%$ \\
$80 \mathrm{~Hz}$ & $27.9 \mathrm{~Hz}$ & $34.8 \%$
\end{tabular}

The maximal value of $f_{1}$, obtained through PSO+ANN optimisation procedure, equals $f_{1}^{P S O}=44.99 \mathrm{~Hz}$ and corresponds to stacking sequence $\boldsymbol{\Lambda}=[81.56 /-37.65 / 15.22 /-$ $1.80 / 5.61 /-1.91 /-7,02]$. The optimisation of $f_{1}$ was performed using also classical optimisation approach, namely interior-point optimisation in Matlab environment, and the obtained result is $f_{1}=44.10 \mathrm{~Hz}$. The results obtained from the proposed PSO+ANN procedure is slightly better and, what is of great importance, obtained using a significantly smaller number of FE calls (and, of course, much faster). Table 1 shows the results of PSO+ANN maximisation of the distance between any natural frequency $f_{i}$ and excitation force frequency $p$. For each of the considered values of excitation frequency, the distance to the nearest natural frequency is higher than $23 \%$ of $p$, what means that the procedure correctly selected the lamination angles and the resonance phenomenon will not appear.

Figure 2 shows the same results like the ones shown in Table 1 in a graphical form. Subsequent rows in Fig. 2 present the results of $f_{1}$ maximisation (the lowest one) and the maximisation of the distance between arbitrarily selected excitation frequencies $(30 \mathrm{~Hz}$ through $80 \mathrm{~Hz}$ ) and neighbouring natural frequencies. The green stripes represent the distance in a frequency space around selected excitation frequency $(30 \mathrm{~Hz}$ through $80 \mathrm{~Hz}$ ) free from natural frequencies, obtained when a particular set of lamination angles is applied. The thick vertical lines represent natural frequencies calculated for the same lamination angles. Although the results, as shown in Table 2, are reasonable from the point of view of resonance avoidance, the upper limit of the frequency gap obtained for $p=30 \mathrm{~Hz}$ smaller than the maximum $f_{1}$ obtained from $f_{1}$ maximisation, this result is incorrect since the stacking sequence obtained 


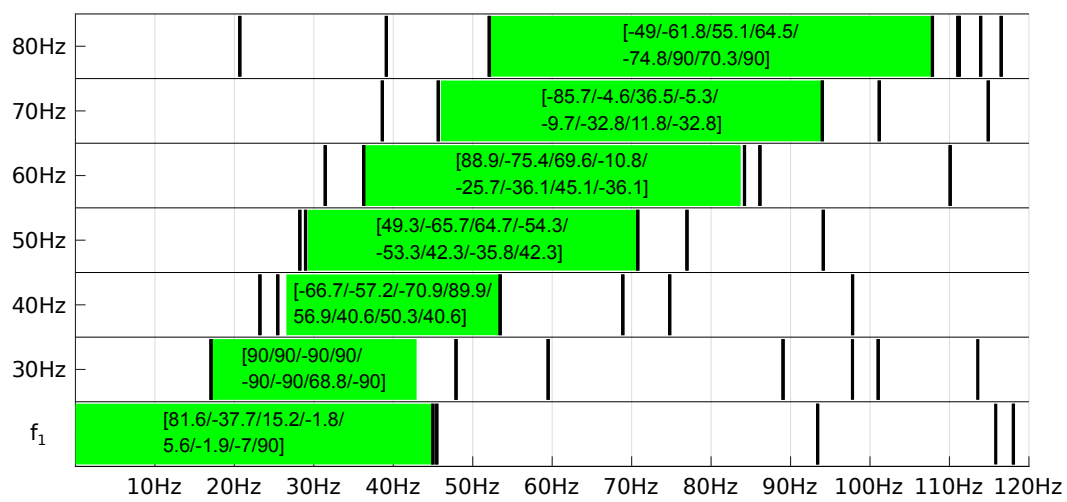

Figure 2. The frequency gap width for different external excitation frequencies

for $f_{1}$ maximisation gives better results (the frequency gap is wider than the one obtained for $p=30 \mathrm{~Hz}$ ) so it is possible to find a better result in this case.

The neural network is thus additionally trained using the new patterns obtained during the first stage of the optimisation described above. Each repetition of the optimisation procedure for each optimisation case is finished with FE verification of the obtained results, the verification data (stacking sequence and corresponding FEM generated natural frequencies) may be treated as an additional example (pattern) to be used during ANN training. Altogether a set of 2,358 patterns are obtained and the ANN is retrained using this pattern set combined with the previous learning pattern set. The overall number of FE calls is increased to 23, 022 , what can be still treated as a very small part of the number of FE calls when the FE is used to calculate the objective function during PSO optimisation.

Table 2. Repeated maximisation of the distance between $f_{i}$ and $p$

\begin{tabular}{ccc}
\hline $\begin{array}{c}\text { External } \\
\text { excitation } \\
\text { frequency }\end{array}$ & \multicolumn{2}{c}{ Minimal distance to $f_{i}$} \\
$1^{\text {st }}$ step & $2^{\text {nd }}$ step \\
\hline$<30 \mathrm{~Hz}$ & $f_{1}^{\text {max }}=44.99 \mathrm{~Hz}$ & $f_{1}^{\text {max }}=45.03 \mathrm{~Hz}$ \\
$30 \mathrm{~Hz}$ & $12.9 \mathrm{~Hz}$ & $15.0 \mathrm{~Hz}$ \\
$40 \mathrm{~Hz}$ & $13.5 \mathrm{~Hz}$ & $13.3 \mathrm{~Hz}$ \\
$50 \mathrm{~Hz}$ & $20.8 \mathrm{~Hz}$ & $20.9 \mathrm{~Hz}$ \\
$60 \mathrm{~Hz}$ & $23.7 \mathrm{~Hz}$ & $24.2 \mathrm{~Hz}$ \\
$70 \mathrm{~Hz}$ & $24.0 \mathrm{~Hz}$ & $24.5 \mathrm{~Hz}$ \\
$80 \mathrm{~Hz}$ & $27.9 \mathrm{~Hz}$ & $28.3 \mathrm{~Hz}$
\end{tabular}

Table 2 and Fig. 3 show the results of the PSO+ANN optimisation performed using retrained ANN, the maximal value of $f_{1}$ equals $f_{1}^{P S O}=45.03 \mathrm{~Hz}$ (corresponding stacking sequence is $\boldsymbol{\Lambda}=[-73.8 / 51.6 / 8.3 /-13.3 / 4.8 / 2.7 /-2.3 / 90])$. The improvement in $f_{1}$ is small, the frequency gaps around excitation frequencies do not raise such doubts as previously described for $p=30 \mathrm{~Hz}$. The results in Fig. 3 show that the investigated $\mathbb{L}^{8}$ space of possible stacking sequences gives, from the point of view of $f_{1}$ and frequency gaps maximisation, a number of solutions similar when considering the result ( $f_{1}$ or frequency gap width) but with different stacking sequence. It is clearly visible for $f_{1}$ maximisation and maximisation of the gap around $p=30 \mathrm{~Hz}$, the dynamic properties of the structure are almost the same, 


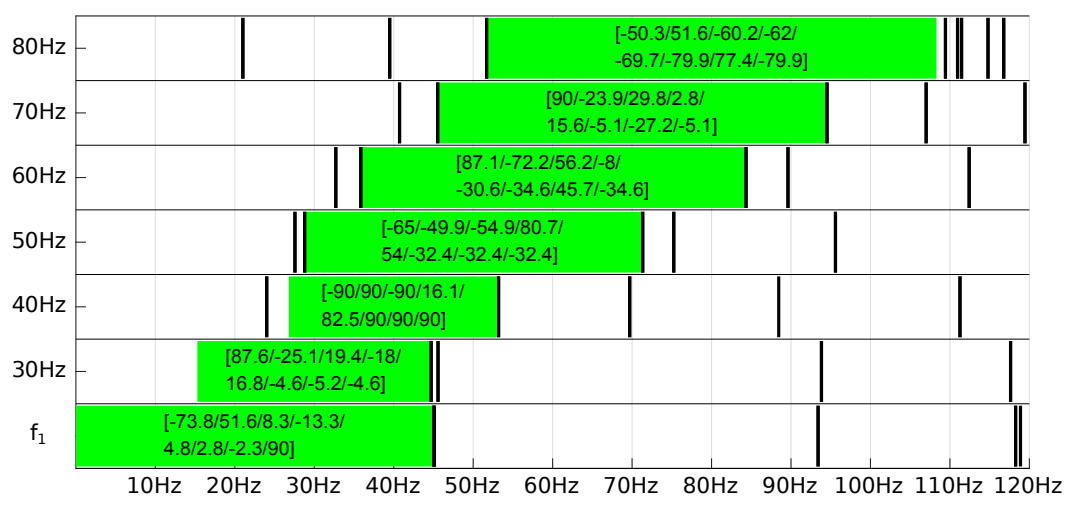

Figure 3. The frequency gap width for different external excitation frequencies ( $2^{\text {nd }}$ step)

while stacking sequences are different $(\boldsymbol{\Lambda}=[-73.8 / 51.6 / 8.3 /-13.3 / 4.8 / 2.8 /-2.3 / 90]$ and $\boldsymbol{\Lambda}=[87.6 /-25.1 / 19.4 /-18 / 16.8 /-4.6 /-5.2 /-4.6]$, respectively). Nevertheless, the proposed PSO+ANN procedure is able to find the expected solution.

\section{Final remarks}

The paper presents a PSO-based method of stacking sequence optimisation in laminated structures. The proposed PSO+ANN procedure can be applied to the optimisation cases where analytical formulae combining design variables and optimised quantities are not possible to derive. The proposed method, due to the replacement of FEM in the calculation of the dynamic parameters of the structure by ANN, accelerates the optimisation and allows to perform the optimisation in a limited time.

The combined PSO+ANN procedure has been successfully applied to problems related to avoiding vibration resonance, which is a major concern for every structure subjected to periodic external excitations. The presented examples show two approaches to the resonance avoidance, through maximisation of the fundamental natural frequency (for external excitation frequencies possibly smaller than the fundamental natural frequency) or through maximisation of a frequency gap around the external excitation frequencies (in other cases).

\section{References}

[1] Bathe Klaus-Jurgen, ADINA: Theory and Modeling Guide. Volume I: ADINA Solids $\mathcal{E}$ Structures (ADINA R\&D, Watertown, 2016)

[2] Chopard Bastien, Tomassini Marco, An Introduction to Metaheuristics for Optimization (Springer, 2018)

[3] Haykin Simon, Neural Networks and Learning Machines (3rd Edition) (Pearson, 2008)

[4] Miller Bartosz, Ziemiański Leonard, Computer Assisted Mechanics and Engineering Sciences 7(4) 781-793 (2000)

[5] Miller Bartosz, Ziemiański Lenoard, Shell Structures: Theory and Applications Volume 4: Proceedings of the 11th International Conference "Shell Structures: Theory and Applications”, (SSTA 2017), October 11-13, 2017, Gdańsk, Poland (CRC Press, Boca Raton 2018)

[6] Vo Thuc Phuong, Lee Jaehong, Ahn Namshik, Composite Structures 89(4) 524-535 (2009) 